\title{
The Turán Number and Probabilistic Combinatorics
}

\begin{abstract}
Alan J. Aw
Abstract

In this short expository article, we describe a mathematical tool called the probabilistic method, and illustrate its elegance and beauty through proving a few well-known results. Particularly, we give an unconventional probabilistic proof of a classical theorem concerning the Turán number $T(n, k, l)$.
\end{abstract}

\section{Introduction.}

Probabilistic combinatorics is a field in combinatorics that was pioneered by one of the greatest mathematicians of all time, Paul Erdős. It utilizes notions from probability theory (mainly discrete probability theory) to prove existence results; for instance, it has provided solutions to the following questions.

- For any graph $G$, can we find a bipartite subgraph which contains at least half of the edges of $G$ ?

- Given a family $\mathcal{F}$ of subsets of $[n]=\{1,2, \ldots, n\}$, if we know its size, can we find two elements $A, B \in \mathcal{F}$ such that $A \subseteq B$ ?

As a matter of fact, we will provide an answer to the second question above.

Mathematicians describe the tools of probabilistic combinatorics using the generic term "the probabilistic method." There is a wide range of techniques in probabilistic combinatorics, and the interested reader should consult [2]. Its diversity notwithstanding, we shall introduce the fundamental ideas of the probabilistic method; perhaps the most straightforward and unambiguous way to do so is to solve a problem. The following theorem, due to Mantel (1907) 4], tells us that in any graph, if it contains many edges, then it contains a triangle. Note that for illustrative purposes, we shall also present a non-probabilistic proof of the theorem.

Theorem 1.1 (Mantel's Theorem) In any graph $G(V, E)$ with $|V|=n$, if $G$ does not contain any triangle, then

$$
|E| \leq\left\lfloor\frac{n^{2}}{4}\right\rfloor
$$


Proof (non-probabilistic). Note: In this proof, we use $\Delta(G)$ to denote the maximum degree among the vertices of $G$.

Label the vertices $v_{1}, v_{2}, \ldots, v_{n}$. Among these vertices, pick a vertex with degree $\Delta(G)$ (there may be more than one such vertex); without loss of generality, let this vertex be $v_{p}$ (for $1 \leq p \leq n$ ). If $\operatorname{deg}\left(v_{p}\right)=k$, then let the set of $k$ neighbours of $v_{p}$ be $P$. If there exists a pair of vertices that are connected by an edge in $P$, then we are done. If not, each vertex in $P$ has degree at most $n-1-(k-1)=n-k$. Moreover, the $n-k-1$ vertices in $V \backslash P$ each have degree at most $k$. Therefore, we have

$$
2|E|=\sum_{i=1}^{n} \operatorname{deg}\left(v_{i}\right) \leq k+(n-k) k+(n-k-1) k=(2 n-2 k) k .
$$

This gives us $|E|=(n-k) k \leq\left(\frac{(n-k)+k}{2}\right)^{2}=\frac{n^{2}}{4}$, where the inequality follows from the Arithmetic-Geometric Mean inequality. Since $|E| \in \mathbb{N}$, we have

$$
|E| \leq\left\lfloor\frac{n^{2}}{4}\right\rfloor
$$

as desired.

Proof (probabilistic). Note: In this proof, a clique on $k$ vertices is simply a set of $k$ vertices in which every two vertices in it are connected by an edge.

Consider a probability distribution $p_{1}, \ldots, p_{n}$ on the vertex set $V$ of $G$. Let us pick two vertices $u, v$ independently and at random (allowing repetition). Then

$$
\mathbb{P}(\{u, v\} \in E)=\sum_{i, j:\{i, j\} \in E} p_{i} p_{j} .
$$

We first assume that the probability distribution is uniform, i.e., $p_{1}=p_{2}=$ $\cdots=p_{n}=\frac{1}{n}$. This gives us

$$
\mathbb{P}(\{u, v\} \in E)=\frac{2|E|}{n^{2}} .
$$

Now, we modify the distribution to make $\mathbb{P}(\{u, v\} \in E)$ as large as possible. This happens when the probability distribution that maximizes the probability is uniform on some maximal clique. Indeed, suppose that there are two nonadjacent vertices $i, j$ such that $p_{i}, p_{j}>0$; let $s_{i}=\sum_{k:\{i, k\} \in E} p_{k}$ and $s_{j}=$ $\sum_{k:\{j, k\} \in E} p_{k}$. If $s_{i} \geq s_{j}$ (resp. $s_{i}<s_{j}$ ), we set the probability of vertex $i$ to $p_{i}+p_{j}$ and the probability of vertex $j$ to zero (and conversely if $s_{i}<s_{j}$ ). This increases $\mathbb{P}(\{u, v\} \in E)$ by $s_{i}\left(p_{i}+p_{j}\right)-\left(p_{i} s_{i}+p_{j} s_{j}\right)=p_{j}\left(s_{i}-s_{j}\right)$ (resp. $\left.p_{i}\left(s_{j}-s_{i}\right)\right)$. 
Since the process is finite, we eventually reach a situation where there are no two non-adjacent vertices of positive probability $\left(p_{i} p_{j}=0\right.$ as long as $\left.\{i, j\} \notin E\right)$, i.e., the probability distribution is on a clique $Q$. Then,

$$
\mathbb{P}(\{u, v\} \in E)=\mathbb{P}(u \neq v)=1-\sum_{i \in Q} p_{i}^{2} .
$$

Assume there is no clique larger than 2 in size (if not, then there would be a triangle violating our assumption). By Jensen's Inequality on the convex function $f(z)=z^{2}$, the above expression is maximized when $p_{i}$ is uniform on $Q$, i.e., $p_{i}=\frac{1}{|Q|}$, which implies

$$
\mathbb{P}(\{u, v\} \in E) \leq 1-\frac{1}{|Q|} \leq 1-\frac{1}{2},
$$

assuming that there is no clique larger than 2 in size (if not there would be a triangle, violating our assumption). Since we started with $\mathbb{P}(\{u, v\} \in E)=\frac{2|E|}{n^{2}}$ and never decreased it in the process, we yield

$$
|E| \leq \frac{n^{2}}{4} \Longrightarrow|E| \leq\left\lfloor\frac{n^{2}}{4}\right\rfloor
$$

as desired.

In fact, Theorem 1.1 can be generalized to the same problem for cliques of size $k$, where $k \geq 3$ (for that matter, Mantel established the result for $k=2$ ). The general problem was solved by the Hungarian mathematician Paul Turán, and a wonderful probabilistic proof of it can be found in [1].

\section{Further Examples.}

Recall the second question in the Introduction regarding the size of a family of sets of $[n]$. Intuitively, if the size of the family, $|\mathcal{F}|$, is large enough, then we should be able to find some $A, B \in \mathcal{F}$ such that $A \subset B$. Indeed, the following theorem of Sperner gives us a sufficient condition on $\mathcal{F}$ such that we can find two sets such that one contains the other.

Theorem 2.1 (Sperner's Theorem) If $\mathcal{F}$ is a Sperner family of subsets (or an antichain) of $[n]$ (i.e., for all $A, B \in \mathcal{F}$ we have $A \not \subset B$ and $B \not \subset A$ ), then

$$
|\mathcal{F}| \leq\left(\begin{array}{c}
n \\
\left\lfloor\frac{n}{2}\right\rfloor
\end{array}\right) .
$$

Proof. Without loss of generality, let $\mathcal{F}=\left\{A_{1}, A_{2}, \ldots, A_{|\mathcal{F}|}\right\}$. Consider any $A_{i} \in \mathcal{F}$ with $\left|A_{i}\right|=a_{i}$. Randomly and uniformly pick any permutation of $[n]$. Denoting by $E_{i}$ the event that the elements in $A_{i}$ appear in the first $a_{i}$ numbers in the permutation, we get 


$$
\mathbb{P}\left(E_{i}\right)=\frac{\left(a_{i}\right) !\left(n-a_{i}\right) !}{n !}=\frac{1}{\left(\begin{array}{c}
n \\
a_{i}
\end{array}\right)} \geq \frac{1}{\left(\begin{array}{c}
n \\
\left\lfloor\frac{n}{2}\right\rfloor
\end{array}\right)} .
$$

Moreover, the $E_{i}$ are mutually exclusive because $E_{i} \cap E_{j}$ is the event that both $A_{i}$ and $A_{j}$ appear at first in the permutation, implying that one of $A_{i} \subset A_{j}$ or $A_{j} \subset A_{i}$ holds. This contradicts our hypothesis. Therefore, summing up our probabilities and noting that probabilities are always less than 1, we obtain

$$
1 \geq \mathbb{P}\left(\bigcup_{i=1}^{|\mathcal{F}|} E_{i}\right)=\sum_{i=1}^{|\mathcal{F}|} \frac{1}{\left(\begin{array}{c}
n \\
a_{i}
\end{array}\right)} \geq \frac{|\mathcal{F}|}{\left(\begin{array}{c}
n \\
\left\lfloor\frac{n}{2}\right\rfloor
\end{array}\right)},
$$

where the last inequality follows from the fact that $\left(\begin{array}{l}n \\ p\end{array}\right)$ is maximized when $p=\left\lfloor\frac{n}{2}\right\rfloor$. The result follows.

Next, we have a classical result that is related to the Turán number $T(n, k, l)$. We shall present a probabilistic proof which differs from the conventional nonprobabilistic proof (see for instance [3, chapter 1]) of the theorem.

Theorem 2.2 The Turán number $T(n, k, l)$, where $n \geq k \geq l$, is the smallest number of l-element subsets of an n-element set $X$ such that every $k$-element subset of $X$ contains at least one of these sets. If $T(n, k, l)=|\mathcal{F}|$, where $\mathcal{F}$ is the smallest family satisfying the above conditions, then

$$
|\mathcal{F}| \geq \frac{\left(\begin{array}{l}
n \\
l
\end{array}\right)}{\left(\begin{array}{l}
k \\
l
\end{array}\right)}
$$

Proof. Let us randomly select without replacement a $k$-element subset $K$ from $X$. Let $A$ be the event that the first $l$ elements picked form a set $L$ which belongs to $\mathcal{F}$. Then

$$
\mathbb{P}(A)=|\mathcal{F}| \cdot\left(\frac{l}{n} \cdot \frac{l-1}{n-1} \cdots \frac{1}{n-l+1} \cdot \frac{n-l}{n-l} \cdots \frac{n-k+1}{n-k+1}\right)=\frac{|\mathcal{F}|}{\left(\begin{array}{c}
n \\
l
\end{array}\right)} .
$$

On the other hand, let us consider the events $E_{i}$ where $E_{i}$ is the event that the $i$ th $k$-element subset was selected. Then we have

$$
\mathbb{P}(A)=\sum_{i=1}^{\left(\begin{array}{c}
n \\
k
\end{array}\right)} \mathbb{P}\left(A \cap E_{i}\right) \geq \sum_{i=1}^{\left(\begin{array}{c}
n \\
k
\end{array}\right)}\left[\frac{1}{\left(\begin{array}{l}
n \\
k
\end{array}\right)} \cdot \frac{1}{\left(\begin{array}{l}
k \\
l
\end{array}\right)}\right]=\frac{1}{\left(\begin{array}{l}
k \\
l
\end{array}\right)},
$$

since each $k$-element subset of $X$ contains at least one element in $\mathcal{F}$. Combining the two equations yields the desired inequality. 


\section{Conclusion}

Truly, the probabilistic method presents a pristine and elegant approach to problem solving. From its very first applications in extremal graph theory by Paul Erdős, to its plethora of applications (see [2, 3]) in coding theory, number theory and geometry, probabilistic combinatorics today has certainly emerged as one of the richest subdisciplines of mathematics where beauty, creativity and rigour converge. In Erdős' own words, "This one's from The Book!"

Acknowledgments. The author would like to thank Professor Sergei Tabachnikov for his comments that helped make several improvements to this article.

\section{References}

[1] Aigner, Martin; Ziegler, Günter M. Proofs from THE BOOK. SpringerVerlag, Berlin, 1999.

[2] Alon, Noga; Spencer, Joel H. The probabilistic method, Second Edition. Wiley-Intersciences Series in Discrete Mathematics and Optimization. Wiley-Interscience, New York, 2000.

[3] Jukna, Stasys Extremal combinatorics: with applications in computer science. Texts in Theoretical Computer Science. An EATCS Series. SpringerVerlag, Berlin, 2001.

[4] Mantel, W. Problem 28, Wiskundige Opgaven 10 (1907). 60-61

Raffles Institution, One Raffles Institution Lane, S575954, Singapore

nalawanij@gmail.com 